As a means of retaining the dressings of a stump, and giving the requisite support at the same time, it has not been found to answer. To explain this, a word as to its mode of applitation. The extremity being elevated by an assistant, and the bandage opened out, the base of the bandage is drawn up under the stump, so as to bring its central point opposite the centre of the triangle; then the corners are brought together on the anterior surface of the $\operatorname{limb}$, and whel the apex of the bandage is carried over the face of the stamp, and laid under the corners, they are tied in a reef-knot, the apex of the bandage being turned over, and pinned down on the knot, or a point a little way below. If these corners are not tied sufficiently tight, the dressings are found not to be retained in their place; whereas, if the corners are tied tightly, greater pressure all round the limb at that point is the result, and a sort of semistrangulated condition of the parts below follows. It was found requisite, therefore, in dressing this sort of cases, to fall kack upon the roller.

I would add a few words on the "küssen" of Stromeyer, for fractures of the upper extremity, and the chloroform bottle and inhaler, but I fear I have taken up too much space already.

Albany-street, April, 1871.

ON THE

\section{THERAPEUTICAL ACTION OF QUININE ON MALARIA.}

\section{Br GOPAUL CHUNDER ROY, F.R.C.S.}

WITH all the boasted researches of modern medicine, the action of quinine on malarious fevers still remains an enigma to solve. The same empiricism which at first introduced this valuable medicine in practice yet actuates the practitioner to prescribe it in blind acknowledgment of its remedial powers. No time, therefore, can be said to be unprofitably spent that is devoted to discuss the topic, How does quinine influence the system? To enable us to comprehend its mode of action, I will take the liberty to advert for a moment to my views on the nature of malaria. It is a subtle poison manifesting itself in certain symptoms which primarily consist of derangement of the functions of the ganglionic nervous centres. The stage of incubation, in which the general malaise, languor, chilliness, and altered secretions, are the marked features of complaint, would afford proof in support of the statement. Nor do the symptoms of actual invasion add less weight to the assertion when we observe the peculiar altered balance of circulation manifested in the cold and hot stages of fever under its influence. The circulatory apparatus of our organism is under the direct control of the sympathetic system; and we can easily understand how an influence exerted over the latter would modify or alter the governing power. The tendency to rapid death by asthenia from the concentrated action of malaria on the system is borne out by experience in the fresh outbreak of an epidemic; and the inflamma. tions of different organs from the same cause, as meningitis, splenitis, hepatitis, dysentery, \&c., are further evidences of the weakening influence of the poison on the local circulation of blood, inducing perverted nutrition in organs probably predisposed to such attacks. When the poison falls short in its virulence, changes in the nutrition of structures occur, which are physiological as far as the process of hypertrophy is concerned, but become of pathological significance on account of their unnatural grow th without any apparent demand or necessity in the economy. Thus the large ague cake, and elephantiasis of the scrotum, leg, or forearm, although they are consistent with perfect health of the individual, yet no doubt are deviations from the natural standard, which, carried beyond an ordinary size, become a source of danger and an incumbrance. To sum up, then : the effect of malaria is primarily spent on the organic system of nerves, especially on the parts governing the circulation and nutrition of our body, producing the varions phases of alterations evidenced in hypertrophy, infainmation, and death, according to its degree of concentration and virulence. It has a tendency to weaken or paralyse the walls of the bloodressels, and favour their dilatation and engorgement.

Quinine is admirably adapted to counteract the influence of malaria by virtue of its tonic effect on the sympathetic system. In enfeebled states of the constitution, it promotes digestion and secretion and gives tone to the heart; under its use the pulse becomes smaller but stronger. In heatapoplexy with great pungency of skin, its exhibition reduces the temperature of the body by counteracting the paralytic condition of the vessels. In intermittent fever, given before the expected paroxysm, it remedies that condition which causes determination of blood to the skin and the consequent pyrexial symptoms. A large dose acts just in the same way as smaller doses repeated at frequent intervals, but with greater energy and certainty. Probably in such instances the former is the more efficient mode of administration; for, inasmuch as the attack ushers itself in a sudden outburst of symptoms, it stands to reason that a stiff dose would be more effective in obviating the tendency. This view will enable us to discard the assumption which ascribed the beneficial action of quinine in such cases to the unknown agency of "shock." It has no peculiar virtue on malarious poison, and, given with the belief that it removes the malarious taint of the constitution, it of ten fails in attaining that end. Hence the notion is gaining ground amongst practitioners that, although quinine will cut short a paroxysm of fever, it does not cure it. Its use can be extended to other diseases where the same defective power is observed, irrespective of malarious origin. Thus in idiopathic and surgical fevers, in burning of the palms of the hands and soles of the feet, and like diseases of perverted circulation, it would prove an invaluable remedy; but when the condition of the part merges into the pathological state of inflammation, as in meningitis or dysentery, I hold its curative power to be at best doubtful.

Glasgow, Feb. 1871.

\section{A CASE OF}

\section{PERFORATING ULCER OF THE STOMACH; RECOVERY.}

\section{BY T. TINLEY, L.R.C.P. EDIN., \&C.}

I AMr induced to forward the particulars of the following case of recovery after perforation of the stomach, it being similar to the one recorded by Dr. Ross, occurring in the North Staffordshire Infirmary, and published in 'l'HE LANCET of Jan. 21st. As I have not any notes of the case, I have to rely entirely on my memory, and can only give the line of treatment pursued, regardless of dates.

I was sent for on Nov. 21st, 1868, to visit Mrs. Bwho had been taken suddenly ill. On arriving at the house I found her in a state of collapse, with a quick, scarcely perceptible pulse, pallid, and covered with cold clammy perspiration. There was constant retching, but no vomiting of any account, merely a small quantity of grumous fluid, having the appearance of blood acted on by the gastric juice. I remained with her some time, and gave her a dose of opium. I found, on inquiry, that for some time she had complained of a burning pain coming on shortly after taking food. The pain was at a fixed point in the epigastric region, shooting through to the back, accompanied by nausea, but only occasional vomiting. She had never sought relief, thinking it would get better. On the morning of the 21st, while preparing dinner, she was suddenly seized with severe pain in the epigastrium, causing her to scream violently, which she continued to do at intervals for some time. Fomentations were applied to the abdomen constantly, and a grain of opium given in pill every third hour, which procured very little rest, and only partially relieved the pain.

On the second day after the occurrence of the above symptoms the pain had extended over the whole abdomen; the knees were drawn up, the pulse became very high and wiry, the countenance anxious, and, in fact, all the symptoms of acute general peritonitis set in. I ordered a turpentine stupe to the abdomen, to be followed by hot fomentations; and beef-tea and brandy injections every fourth hour. Neither liquid nor solid was taken into the stomach for 